\title{
Study on Abelian dominance of Interquark potential in SU (3) Lattice QCD using simulation Techniques
}

\author{
Chitra Kandpal, Sukhwinder Singh and Devraj Mishra \\ Department of Physics, R H Govt P G College; Kashipur U S Nagar-262401 \\ Email: chitrakandpal700@gmail.com
}

\begin{abstract}
It is assumed that no free quark to be found in nature. This is known as the confinement problem, is an issue which has no analytic proof till yet. But lattice QCD Monte Carlo simulations have shown that for large distances the quark antiquark potential varies linearly with interquark distance and controlled by the string tension. In the present work we have revisited the study of interquark potentials and Abelianization of QCD in Maximally Abelian gauge in context of dual superconducting picture. We have also observed the perfect Abelian dominance of the string tension for various spatial size lattices.
\end{abstract}

Keywords: Quark confinement, Abelian dominance, Lattice QCD.

\section{Introduction}

The simulation calculations are made at a large distance scale to understand the phenomenon of quark confinement. Mathematically interquark potential V(r) [1] is given as-

$$
\mathrm{V}(\mathrm{r})=-\frac{\mathrm{A}}{\mathrm{r}}+\sigma \mathrm{r}+\mathrm{C}
$$

where, $\mathrm{r}$ is the interquark distance, and $\sigma$ is the string tension, $\mathrm{A}$ is color-Coulomb coefficient and $\mathrm{C}$ is an irrelevant constant. Lattice QCD study gives that the linear confinement term arises due to the one dimensional color flux squeezing of the quarks [1]. The dual superconducting picture [2] provides a framework for the study of quark confinement. To establish dual superconductor picture, a mathematical procedure called Abelian Projection has been proposed by t'Hooft [3] through which the confining physics of SU (3) QCD can be reduce to an Abelian U (1) X U (1) gauge theory including monopole degrees of freedom. The gluon field $A_{\mu}$ in QCD has both diagonal and off diagonal parts. In continuum, Cartan decomposition is given by -

$$
A_{\mu}=\bar{A}_{\mu^{*}} \bar{H}+\sum_{\alpha} A_{\mu}^{\alpha} T_{\alpha}
$$

where, $\bar{H}=T_{3}, T_{8}$ are the diagonal generators, and $T_{\alpha=1,2,4,5,6,7}$ are the off diagonal generators of SU(3).The off diagonal part $\Sigma_{\alpha} A_{\mu}^{\alpha} T_{\alpha}$ represent the non abelian nature and $A_{\mu} \rightarrow \bar{A}_{\mu}, \bar{H}$ induces the Abelian Projection. For MA projection [4,5] the minimization of off diagonal part is given as-

$R_{\text {off }}\left[A_{\mu}(x)\right] \equiv \int d^{4} x \sum_{\mu, \alpha}\left|A_{\mu}^{\alpha}(x)\right|^{2}$

The SU(3) QCD link variables $U_{\mu}(s)$ is given as-

$$
U_{\mu}(s)=e^{i a g A_{\mu}(s)} \in S U(3)
$$

where ' $a$ ' is the lattice spacing and ' $g$ ' is gauge coupling.

The Abelian part of the link variable is extracted as-

$$
\begin{aligned}
& \left.u_{\mu}(\mathrm{s})=\exp \left(\mathrm{i} \theta_{\mu}^{3}(\mathrm{~s}) \mathrm{T}_{3}+\mathrm{i} \theta_{\mu}^{8}(\mathrm{~s})\right) \mathrm{T}_{\mathrm{g}}\right) \\
& \in U(1)_{3} \times U(1)_{8}
\end{aligned}
$$

and the off diagonal link variable is defined as- 
$M_{\mu}(s)=\mathrm{U}_{\mu}^{\mathrm{MA}}(\mathrm{s}) u_{\mu}^{\dagger}(s) \in S U(3) / U(1)_{3} \times$
$U(1)_{8}$

(6)

which leads to the Cartan decomposition of the gauge group

$$
\begin{gathered}
\mathrm{U}_{\mu}^{\mathrm{MA}}(s)=M_{\mu}(s) u_{\mu}(\mathrm{s})= \\
\mathrm{e}^{\mathrm{i} \sum \theta_{\mu}^{\alpha} \mathrm{T}_{\alpha}(\mathrm{s})} \mathrm{e}^{\mathrm{i} \theta_{\mu}(\mathrm{s}) \cdot \mathrm{H}} \in S U(3)
\end{gathered}
$$

\section{Numerical Formalism}

We review the numerical analysis performed by N. Sakumichi et.al [6] for $\mathrm{Q} \bar{Q}$ potential calculations.

For a closed $\mathrm{R} \times \mathrm{T}$ rectangle trajectory $\mathrm{C}$, the Wilson loop is given as-

$$
W_{C}\left[U_{\mu}(s)\right] \equiv \operatorname{Tr}\left[\Pi_{c} U_{\mu}(s)\right]
$$

The quark-antiquark potential was calculated as-

$$
V(r)=-\lim _{T \rightarrow \infty} \frac{1}{T} \ln \left\langle W_{C}\left[U_{\mu}(s)\right]\right\rangle
$$

where $(\ldots)$ is the statistical average over the gauge configuration. The MA projection of the quark-antiquark potential was calculated as-

$$
V_{A b_{\theta l} l}(r)=-\lim _{T \rightarrow \infty} \frac{1}{T} \ln \left\langle W_{C}\left[u_{\mu}(s)\right]\right\rangle
$$

The off diagonal part of the quark-antiquark potential was calculated as-

$$
V_{\text {off }}(r)=-\lim _{T \rightarrow \infty} \frac{1}{T} \ln \left\langle W_{C}\left[M_{\mu}(s)\right]\right\rangle
$$

\section{Results}

Fig.1 reflects that at large distances there is resemblance in the behaviour of $\mathrm{V}(\mathrm{r})$ and $V_{A b_{e l} l}(r)$. The same slope of $\mathrm{V}(\mathrm{r})$ and $V_{A b e l}(r)$ suggests the Abelian dominance for confinement. From the figure it appears that $\mathrm{V}(\mathrm{r})-V_{A b e l}(r)=V_{\text {off }}(r)$ is well described by pure Coulomb ansatz and have almost zero string tension $\sigma \sim 0$.

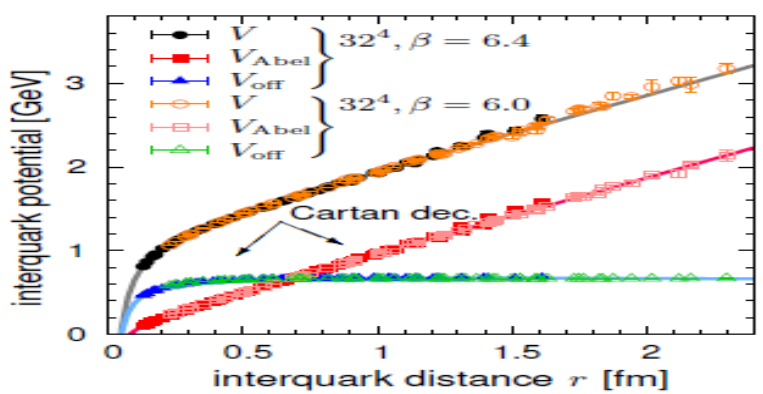

Figure 1 (Color online): Cartan decomposition of the $\mathrm{Q} \bar{Q}$ potential.

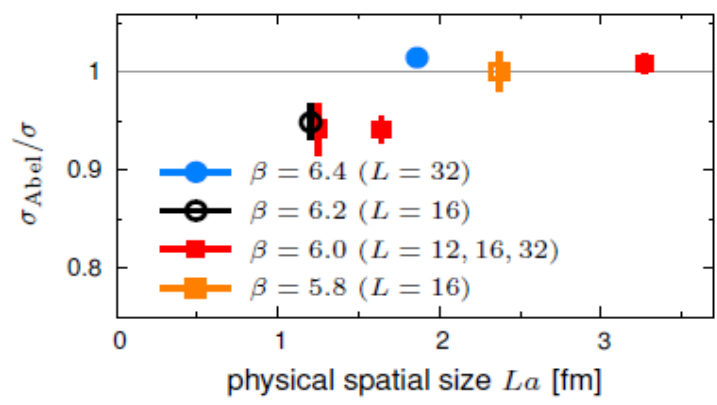

Figure 2 (Color online): Represent Physical spatial-size dependence of $\boldsymbol{\sigma}_{\text {abel }} / \boldsymbol{\sigma}$.

Phys.Rev.D90, 11501(R) (2014)

Fig. 2 reflects that for the smaller lattices only approximate Abelian dominance was observed. Perfect Abelian dominance seems to be realized when spatial size $\mathrm{La}$ is sufficiently large [6].

\section{Acknowledgements}

Authors would like to thank to Principal of RHGPG College Kashipur and Department of Physics, RHGPG College Kashipur for their generous support in perusing these studies. 


\section{References}

[1] H. J. Rothe, Lattice Gauge Theories, 4th ed. (World Scientific, Singapore, 2012) [2] Y. Nambu, Phys. Rev. D 10, 4262 (1974); G.'t Hooft, in High Energy Physics; S. Mandelstam, Phys. Rep. 23, 245 (1976) [3] G. 't Hooft, Nucl. Phys. B190, 455 (1981) [4] K. Amemiya and H. Suganuma, Phys. Rev. D 60, 114509(1999)

[5] J. D. Stack, S. D. Neiman, and R. J.

Wensley, Phys. Rev.D 50, 3399 (1994).

[6] Naoyuki Sakumichi and Hideo Suganuma, Phys.Rev.D90, 11501(R) (2014). 